\title{
Máxima Información, Mínima Molestia (MIM0): Reducir cuestionarios para cuidar de las personas participantes en psicología del deporte.
}

\section{Maximum Information, Minimum Discomfort (MIMO). Shortening questionnaires to take care of participants in sport psychology}

Fecha de recepción: 22-01-2020

Fecha de aceptación: 18-06-2020

\author{
Saul Alcaraz* \\ Anna Jordana* \\ Joan Pons* \\ Marta Borrueco* \\ Yago Ramis* \\ Miquel Torregrossa* \\ *Sport Research Institute UAB, Universitat Autònoma de Barcelona
}

\section{resumen/ahstract:}

Contestar a un gran número de ítems puede suponer una gran carga para las personas participantes, lo cual puede empeorar la calidad de los datos recogidos. Por este motivo, el propósito de nuestro estudio es presentar el método de Máxima Información, Mínima Molestia (MIMO) para el desarrollo de versiones cortas en psicología del deporte. Nuestro protocolo propone cuatro fases para preparar la versión corta de un cuestionario ya existente. Concretamente, proponemos (a) realizar una selección inicial de ítems en base a criterios teóricos, psicométricos y de comprensión (Fase I); (b) refinar la versión corta gracias a las opiniones de un panel de personas expertas (Fase II); (c) evaluar la comprensión de los ítems por parte de la población diana (Fase III); y (d) evaluar la estructura factorial, fiabilidad y validez de relación con otras variables (Fase IV). Para cada fase, aportamos justificación, explicamos su desarrollo, presentamos las personas involucradas y ofrecemos un ejemplo de caso práctico de cómo podría aplicarse. En esencia, el método MIMO permite desarrollar versiones cortas que cuiden de las personas participantes, evalúen los aspectos clave de los constructos, conserven las propiedades psicométricas e incrementen la calidad de los datos recogidos.

Responding to a large number of items could be a great burden for the participants, which can worsen the quality of the data collected. Therefore, the purpose of the present study is to describe the method of Maximum Information, Minimum Discomfort (MIMO in Spanish) to develop questionnaire short-forms in sport psychology. Our protocol suggests four phases to create the short-form of an existing questionnaire. Specifically, we recommend: (a) selecting an initial pool of items based on theory-driven, data-driven and comprehension-related criteria (Phase l), (b) refining the short-form with the evaluation of a panel of experts (Phase II), (c) assessing target population's understanding of the items (Phase III), and (d) evaluating factor structure, reliability and validity evidence based on relation to other variables of the short-form (Phase IV). We describe each phase (i.e., justification, procedure and participants involved) and then we present a real case showing how these phases could be carried out. In sum, the MIMO method explains how to develop short-forms that take care of participants, assess the core of constructs, minimize the loss of psychometric properties and increase the quality of the data collected.

\section{palabras clave/keywords:}

Cuestionario, calidad de los datos, versiones cortas de cuestionarios, refinamiento de cuestionarios, desarrollo de cuestionarios.

Questionnaire, data quality, questionnaire short-forms, questionnaire refinement, questionnaire development 
Los estudios en psicología del deporte han ido necesitando progresivamente un mayor número de ítems a medida que se ha ido profundizando en el contenido de las teorías y en las relaciones entre constructos, lo cual ha aumentado necesariamente el tiempo que los y las participantes destinan a rellenar cuestionarios (Alcaraz, Viladrich, y Torregrossa, 2013). En ese sentido, incrementar el tiempo de respuesta supone una carga mayor para las personas participantes (e.g., aburrimiento, cansancio) y puede conllevar una pérdida de calidad en los datos recogidos (e.g., valores perdidos, menor tasa de respuesta, mayor tasa de abandono en estudios longitudinales, aumento del error de medida; Maloney, Grawitch, y Barber, 2011; Yan, Fricker, y Tsai, 2020). Complementariamente, tanto en la investigación aplicada como en el asesoramiento a deportistas, se ha subrayado la necesidad de disponer de instrumentos cortos y comprensibles, al mismo tiempo que válidos y fiables (Horvath y Röthlin, 2018). Asimismo, tal y como indican estos autores, los y las profesionales necesitan instrumentos que les permitan realizar las tareas de cribado y monitorización de una forma eficiente. Ante esta situación, las versiones cortas de cuestionarios ya existentes pueden ayudar a solventar estas problemáticas, dado que reducen el número de ítems totales, al mismo tiempo que disminuyen la fatiga de las personas participantes e incrementan la calidad de los datos (e.g., Little, Jorgensen, Lang, y Moore, 2014).

El cuidado de las personas participantes, tanto en la investigación como en el asesoramiento, es de suma relevancia. Su tiempo es limitado y, tal como indican Horvath y Röthlin (2018), es necesario imponer la sensatez: Un mayor número de ítems puede mejorar la validez y fiabilidad de los instrumentos, pero al mismo tiempo perjudica la ratio entre el esfuerzo de las personas participantes y los beneficios para la investigación. Por ejemplo, se puede eliminar la repetitividad de ciertos ítems, lo cual ayudará a disminuir la fatiga de las personas que contestan el cuestionario (Moore, Brown, y Fry, 2015). Paralelamente, disponer de medidas cortas permitirá una evaluación más rápida, que pueda adaptarse a las necesidades y situaciones de las personas que contestan el instrumento (e.g., antes de los entrenamientos).

A pesar de los beneficios que puede conllevar el desarrollo de versiones cortas, en el ámbito de la psicología del deporte éste es un campo todavía poco explorado (Alcaraz et al., 2013). De hecho, los resultados de una búsqueda realizada a principios del año 2020 en las bases de datos PsycInfo, SPORTDiscus y Web of Science con la combinación de palabras clave sport psycholog*, develop*, short-form*, shorten*, questionnaire*' e instrument ${ }^{*}$ mostraron que únicamente 10 artículos habían tenido como objetivo presentar el desarrollo de una versión corta en el periodo 2008-2019. La búsqueda puso también de manifiesto que cada uno de estos artículos planteaba formas distintas de desarrollar las versiones cortas, tanto en relación con el procedimiento planteado como con los criterios utilizados para realizar la selección de los ítems.

Una posible explicación para este bajo número de artículos es que a veces su publicación es difícil, porque reducir el número de ítems por escala puede dificultar el cumplimiento de los criterios de calidad de la investigación básica (Horvath y Röthlin, 2018). Otra explicación plausible se deriva de los resultados de la búsqueda comentada anteriormente, dado que señalaron una falta de publicaciones que explicaran de forma detallada cómo preparar y llevar 
a cabo el desarrollo de versiones cortas en psicología del deporte. Esto, en consecuencia, puede dificultar que los investigadores e investigadoras dispongan del conocimiento y/o de las habilidades necesarias para llevar a cabo el desarrollo de versiones cortas, a pesar de que puedan conocer sus ventajas.

Además de ayudar a reducir la carga impuesta en las personas respondientes, es necesario remarcar que las versiones cortas permiten ser eficientes en dos fases clave de la investigación: la creación de instrumentos y la recogida de datos. Por un lado, tal como señalaron Coste, Guillemin, Pouchot y Fermanian (1997), crear una versión corta permite ahorrarse algunas de las fases del desarrollo de un nuevo cuestionario y facilita el uso de la nueva versión, dado que ésta les será familiar a aquellas personas que ya habían utilizado el cuestionario completo. Es decir, generar una versión corta es preferible dado que es un procedimiento más eficiente en cuanto al desarrollo del instrumento.

Por otro lado, debemos tener en cuenta la eficiencia en la fase de recogida de datos. En palabras de Mülhan, Bullinger, Power y Schmidt (2008), la eficiencia de una versión corta hace referencia a la comparación entre la economía (i.e., longitud del cuestionario reducida o tiempo ahorrado con la versión corta) y la pérdida de información o validez. En ese sentido, tal como señalaron Smith, McCarthy y Anderson (2000), todo proceso de reducción o refinamiento de instrumentos puede conllevar una pérdida de propiedades psicométricas. En estas circunstancias, el reto es poder plantear un proceso reducción de cuestionarios que permita minimizar estas pérdidas al mismo tiempo que permita obtener datos de mejor calidad.

Teniendo en cuenta la necesidad de disponer de un protocolo que facilite el desarrollo y validación de versiones cortas de cuestionarios ya existentes en psicología del deporte, el propósito de este estudio es presentar el método de Máxima Información, Mínima Molestia (MIMO). Tal como indica su propio nombre, este modelo detalla cómo desarrollar versiones cortas que retengan la información clave de los constructos al mismo tiempo que disminuyan la carga en las personas participantes (e.g., tiempo, esfuerzo) y minimicen la pérdida de propiedades psicométricas. Considerando la falta de publicaciones que detallen cómo llevar a cabo la creación de una versión corta, hemos centrado nuestro trabajo en el aspecto procedimental, en el cómo hacerlo. Concretamente, en primer lugar, presentamos una visión global del modelo y a continuación detallamos las cuatro fases que forman parte de éste, incluyendo su justificación, proceso de aplicación y participantes involucrados. Complementariamente, ofrecemos un ejemplo de caso práctico para ilustrar cómo puede aplicarse el método MIMO para desarrollar una versión corta de un cuestionario perteneciente al ámbito de la carrera dual (i.e., combinación del deporte y la educación).

\section{Método MIMO: Máxima Información, Mínima Molestia}

\section{Visión general del método MIMO}

Mediante el método MIMO planteamos un proceso de cuatro fases sucesivas para la preparación y validación de la versión corta de un cuestionario ya existente (en la Figura 1 puede observarse el desarrollo global del método MIMO). Concretamente, en la Fase I 

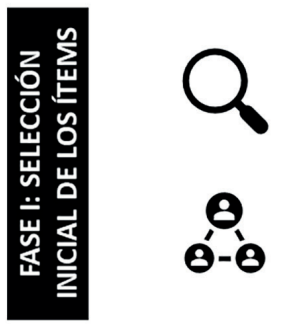

$$
\begin{gathered}
\text { Obtención de criterios } \\
\text { teóricos, psicométricosy } \\
\text { de comprensión }
\end{gathered}
$$
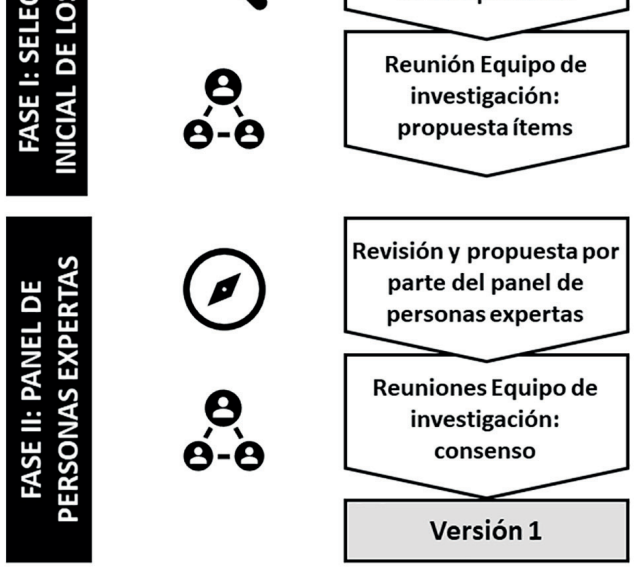

Reuniones Equipo de investigación: consenso
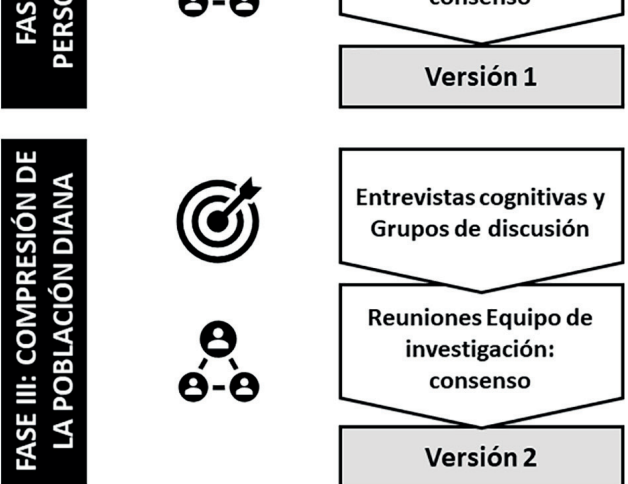

Entrevistas cognitivas y Grupos de discusión
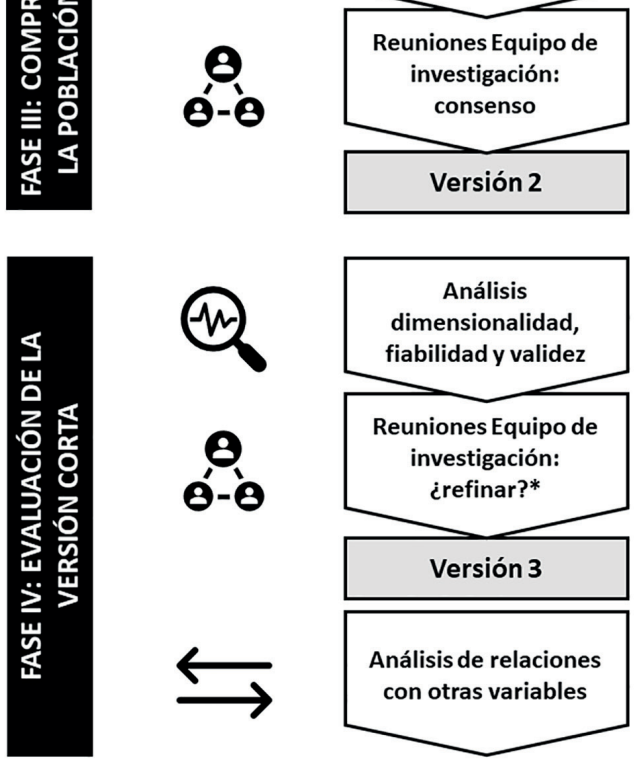

Figura 1.- Visión general del método MIMO (Máxima Información, Mínima Molestia) para la preparación de versiones cortas en psicología del deporte.

El formato de esta figura se ha inspirado en la presentada por Boateng et al. (2018).

Nota. *Esta fase se llevará a cabo únicamente si se estima necesaria de acuerdo con los resultados de los análisis de dimensionalidad, fiabilidad y validez. 
proponemos realizar la selección inicial de los ítems del cuestionario original que pasarían a formar parte de la versión corta, en base a criterios teóricos, psicométricos y de comprensión. En la Fase II, sugerimos llevar a cabo un refinamiento de la versión corta mediante un panel de personas expertas. Fruto de esta fase se obtendría la primera versión del cuestionario reducido (Versión 1). En la Fase III, evaluaríamos la comprensión de los ítems por parte de la población diana, mediante la recogida de datos cualitativos. Y, finalmente, en la Fase IV planteamos realizar una recogida de datos que permita analizar las propiedades psicomé-

\section{Fase I: Selección inicial de los ítems}

Objetivo: realizar la selección inicial de ítems en base a criterios teóricos, psicométricos y de comprensión

\begin{tabular}{|l|l|l}
\hline Actividad & \multicolumn{1}{c|}{ Desarrollo (aspectos clave) } & \multicolumn{1}{c}{ Participantes } \\
\hline $\begin{array}{l}\text { Obtención de criterios teóricos, } \\
\text { psicométricos y de comprensión }\end{array}$ & $\begin{array}{l}\text { Buscar fuentes de información para los tres } \\
\text { criterios de selección de los ítems (e.g., bases de } \\
\text { datos, búsqueda bibliográfica). }\end{array}$ & $\begin{array}{l}\text { Equipo de investigación común para todo el } \\
\text { proceso: Conocimiento del constructo, } \\
\text { experiencia en el desarrollo de instrumentos y en } \\
\text { metodologías cualitativas. }\end{array}$ \\
\hline $\begin{array}{l}\text { e-e } \\
\text { Reuniones Equipo de }\end{array}$ & $\begin{array}{l}\text { Poner en común la información. Priorizar los } \\
\text { criterios de contenido sobre los psicométricos. }\end{array}$ & $\begin{array}{l}\text { Equipo de investigadores/as común para todo el } \\
\text { proceso }\end{array}$
\end{tabular}

\section{Fase II: Panel de personas expertas}

Objetivo: refinar la versión corta gracias a las opiniones de un panel de expertos/a

\begin{tabular}{ll|l|l}
\hline Actividad & \multicolumn{1}{|c}{ Desarrollo (aspectos clave) } & \multicolumn{1}{c}{ Participantes } \\
\hline $\begin{array}{l}\text { Revisión y propuesta por parte } \\
\text { del comité de expertos/as }\end{array}$ & $\begin{array}{l}\text { Pedir a las personas expertas que evalúen la } \\
\text { relevancia de los ítems, detecten los ítems a } \\
\text { eliminar y aporten argumentación cualitativa. }\end{array}$ & $\begin{array}{l}5-7 \text { personas expertas en los conceptos } \\
\text { evaluados por la versión corta. Independientes } \\
\text { del equipo de investigación }\end{array}$ \\
\hline $\begin{array}{lll}\text { Reuniones Equipo de } \\
\text { investigación: consenso }\end{array}$ & $\begin{array}{l}\text { Evaluar la concordancia entre expertos/as. } \\
\text { Revisar su argumentaciones. }\end{array}$ & $\begin{array}{l}\text { Equipo de investigación común para todo el } \\
\text { proceso. }\end{array}$
\end{tabular}

\section{Fase III: Comprensión de la población diana}

Objetivo: evaluar la comprensión de los ítems por parte de la población diana

\begin{tabular}{|l|l|l}
\hline Actividad & \multicolumn{1}{|c|}{ Desarrollo (aspectos clave) } & \multicolumn{1}{c}{ Participantes } \\
\hline $\begin{array}{l}\text { C) Entrevistas cognitivas y Grupos } \\
\text { de discusión }\end{array}$ & $\begin{array}{l}\text { Analizar la comprensión de los ítems. Animar a } \\
\text { compartir alternativas de redactado. }\end{array}$ & $\begin{array}{l}\text { Personas de la población diana. Incluir aquellas } \\
\text { subgrupos con más dificultades de comprensión. }\end{array}$ \\
\hline $\begin{array}{l}\text { O-e Reuniones Equipo de } \\
\text { investigación: consenso }\end{array}$ & $\begin{array}{l}\text { Discutir las propuestas de la población diana. } \\
\text { Incluir aquéllas que mejoren la comprensión de } \\
\text { los ítems sin modificar el significado. }\end{array}$ & $\begin{array}{l}\text { Equipo de investigación común para todo el } \\
\text { proceso. }\end{array}$ \\
\hline
\end{tabular}

\section{Fase IV: Evaluación de la versión corta}

Objetivo: evaluar la dimensionalidad, fiabilidad y validez de la versión corta y su relación con otras variables

\begin{tabular}{|c|c|c|c|}
\hline \multicolumn{2}{|c|}{ Actividad } & Desarrollo (aspectos clave) & Participantes \\
\hline (4). & $\begin{array}{l}\text { Análisis dimensionalidad, } \\
\text { fiabilidad y validez }\end{array}$ & $\begin{array}{l}\text { Realizar los análisis factoriales confirmatorios, } \\
\text { análisis de invariancia y de consistencia interna. }\end{array}$ & Personas de la población diana. \\
\hline ô & $\begin{array}{l}\text { Reuniones Equipo de } \\
\text { investigación: ¿̇refinar?* }\end{array}$ & $\begin{array}{l}\text { Detectar los ítems con rendimiento pobre. } \\
\text { Analizar la posibilidad de introducir cambios. }\end{array}$ & $\begin{array}{l}\text { Equipo de investigación común para todo el } \\
\text { proceso. }\end{array}$ \\
\hline$\leftrightarrows$ & $\begin{array}{l}\text { Análisis de relaciones con otras } \\
\text { variables }\end{array}$ & $\begin{array}{l}\text { Analizar las relaciones esperadas con otras } \\
\text { variables. Comparar con la versión original. }\end{array}$ & Personas de la población diana. \\
\hline
\end{tabular}

Figura 2.- Presentación de las cuatro fases del Método de Máxima Información y Mínima Molestia (MIMO).

Nota. *Esta fase se llevará a cabo únicamente si se estima necesaria de acuerdo con los resultados de los análisis de dimensionalidad, fiabilidad y validez. 
tricas de la versión corta. En la Figura 2 se presentan los aspectos clave de cada una de estas fases.

Para el planteamiento del método MIMO, hemos tenido en cuenta el desarrollo de versiones cortas en psicología del deporte (e.g., Milavic et al., 2019; Moore et al., 2015), las directrices para el desarrollo y validación de cuestionarios (e.g., AERA, APA, y NCME, 2014; Boateng, Neilands, Frongillo, Melgar-Quiñonez, y Young, 2018) y los criterios habitualmente aceptados para la creación de versiones cortas de cuestionarios ya existentes (Maïano et al., 2008; Marsh, Ellis, Parada, Richards, y Heubeck, 2005; Smith et al. 2000). Estos últimos autores señalan que la nueva versión corta debería: (a) cubrir el mismo contenido en cada factor que el instrumento original, (b) retener la misma estructura factorial, (c) mostrar una estructura factorial con un ajuste satisfactorio a los datos, (d) mostrar una adecuada fiabilidad y (e) relacionarse con otras variables de la misma forma que el cuestionario original.

Complementariamente, es necesario señalar tres aspectos primordiales a la hora de poner en marcha el método MIMO. En primer lugar, es necesario seleccionar un cuestionario con propiedades psicométricas contrastadas para generar su versión corta (Maïano et al., 2008; Marsh et al., 2005; Smith et al., 2000). De esta forma, tendremos un punto de vista sólido sobre el que desarrollar los supuestos que debe cumplir la versión corta (e.g., número de factores esperados, relaciones entre factores).

En segundo lugar, es necesario plantearse hasta qué punto se debería reducir un cuestionario. Estudios anteriores en psicología del deporte han preparado versiones cortas con hasta tres ítems por factor (e.g., Vlachopoulos, Leptokaridou, y Fox, 2014). Sin embargo, los trabajos de Watson y Clark (1997), y Jokovic, Locker y Guyatt (2006) sugirieron un mínimo de cuatro ítems por factor, dado que una mayor reducción conllevaría una consistencia interna insuficiente. Este criterio se ha aplicado en investigaciones posteriores en psicología del deporte, con resultados satisfactorios (e.g., Alcaraz et al., 2013).

Por último, sugerimos conjuntar un equipo de investigación que acompañe toda la aplicación del método MIMO. Como puede observarse en la Figura 1, el equipo de investigación juega un papel fundamental en todas las fases, dado que tiene la tarea de realizar la primera selección de ítems y la responsabilidad de consensuar el cierre de cada una de las fases del método MIMO. Manteniendo los mismos integrantes del equipo nos aseguraremos de que los criterios de funcionamiento interno y de selección de ítems se mantienen consistentes, lo cual facilitará una toma de decisiones coherente a lo largo de todo el proceso. Para completar la información sobre este equipo de investigación, en la Fase I se explican los perfiles de las personas que podrían formar parte de este grupo.

A continuación, detallamos las cuatro fases del método MIMO, incluyendo recomendaciones para su desarrollo y para la selección de las personas participantes en cada fase. Paralelamente, para ayudar a la comprensión del protocolo propuesto, ofrecemos un ejemplo de aplicación del método MIMO para reducir el Cuestionario de Competencias para la Carrera Dual de Deportistas (DCCQ-A, por sus siglas en inglés; De Brandt et al., 2018). El DCCQ-A permite examinar la importancia y la posesión que otorgan los deportistas a las competencias para la carrera dual, y por lo tanto es un instrumento de gran utilidad tanto en 
la investigación básica y aplicada como en el asesoramiento a deportistas. El cuestionario consta de 29 ítems repartidos en cuatro factores: (1) Gestión de la carrera dual, (2) Planificación de carrera, (3) Conciencia emocional e (4) Inteligencia social y adaptabilidad. Los ítems del DCCQ-A se contestan en una escala tipo Likert de cinco puntos. En base al método MIMO, explicamos el proceso seguido para reducir el cuestionario original de 29 ítems a la versión corta de 16 ítems, con cuatro ítems por escala. Tal como puede observarse en la explicación del caso práctico, hemos optado por describir el desarrollo de la versión corta de forma general, con el propósito de ayudar a entender las fases del método MIMO, pero sin entrar en detalles que hubieran dificultado la fluidez general del trabajo.

\section{Fase I: Selección inicial de los ítems}

\section{Desarrollo de la Fase I}

En esta fase, el propósito es llevar a cabo una primera selección de ítems, en base a criterios teóricos, psicométricos y de facilidad de comprensión. Los criterios teóricos se enfocan sobre todo a seleccionar aquellos ítems que hagan referencia al núcleo del constructo medido por la escala. Por su parte, los criterios psicométricos se relacionan, principalmente, con los estadísticos descriptivos de los ítems y con la validez relacionada con la estructura interna. Es necesario señalar que los criterios psicométricos pueden dividirse en dos: aquellos provenientes de datos propios, en el caso de que se disponga de datos obtenidos con la versión larga del cuestionario, y aquellos obtenidos de la literatura previa. En relación con el segundo tipo (ver Maloney et al., 2011), recomendamos realizar una búsqueda bibliográfica de aquellos artículos que hayan utilizado el cuestionario original y detectar los ítems que sistemáticamente hayan ofrecido un rendimiento pobre (i.e., aquellos ítems eliminados de los análisis a causa de sus propiedades psicométricas). Por último, los criterios de facilidad de comprensión pretenden anticipar que los ítems seleccionados sean entendidos correctamente por la población diana (Horvath y Röthlin, 2018). A modo de resumen, en la Figura 3 se muestran los criterios que proponemos tener en cuenta en esta fase.

Aunque algunos trabajos hayan utilizado únicamente criterios psicométricos para el desarrollo de sus versiones cortas (e.g., Noetel, Ciarrochi, Sahdra, y Lonsdale, 2019), sugerimos tener en cuenta los tres tipos de criterios, y no únicamente uno o dos de ellos. Esto permitirá seleccionar ítems teóricamente relevantes, fuertes psicométricamente y de fácil comprensión para la población diana. Complementariamente, al igual que otros autores (e.g., Alcaraz et al., 2013; Coste et al., 1997), recomendamos priorizar los criterios basados en el contenido de los ítems (i.e., teóricos y de comprensión) por encima de los psicométricos.

En relación con el planteamiento de la Fase I, se deben tener en cuenta principalmente dos tareas. Por un lado, será necesario recabar toda la información posible en base a los tres criterios anteriores. Esto implicará la ejecución de las búsquedas en bases de datos y la realización de los análisis de datos, en el caso de disponer de datos del cuestionario original. Por el otro, se deberán plantear reuniones del equipo de investigación dirigidas a revisar los argumentos recabados para llegar al consenso sobre los ítems identificados como problemáticos, que en consecuencia deban ser eliminados (i.e., por su pobre aportación teórica, sus propiedades psicométricas insuficientes y/o su dificultad de comprensión). En 


\section{Criterios teóricos}

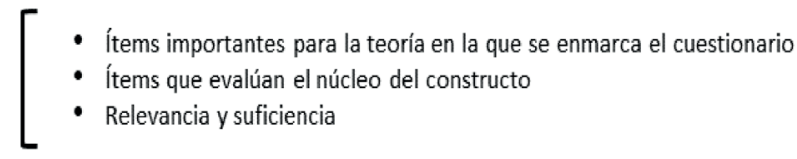

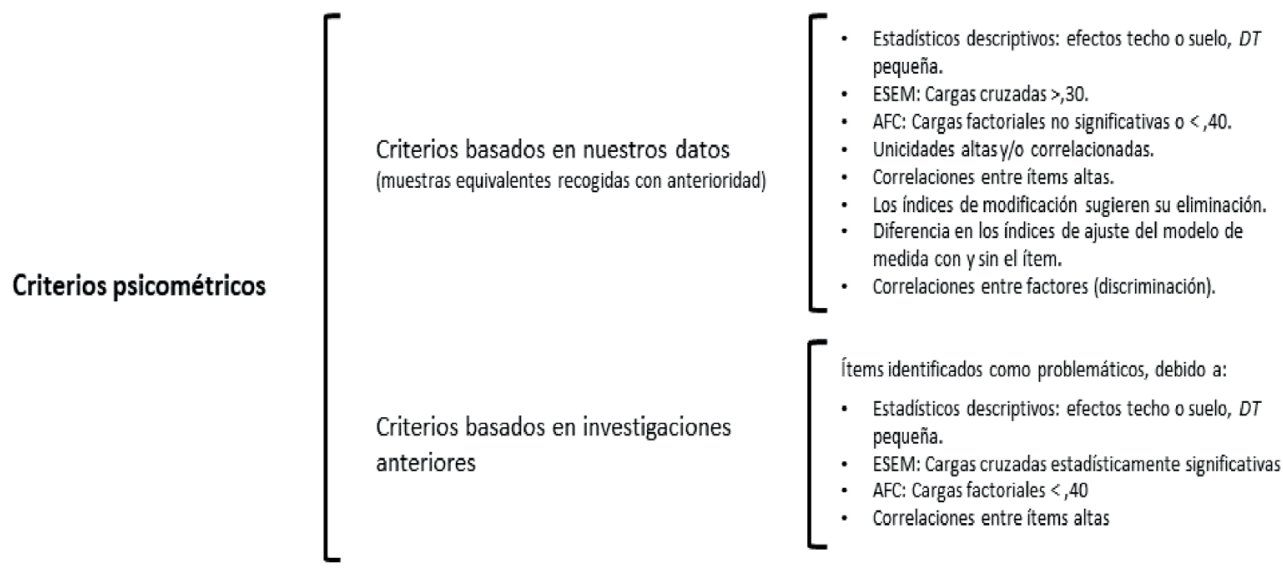

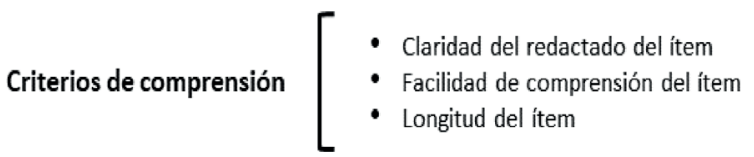

Figura 3.- Criterios teóricos, psicométricos y de comprensión para seleccionar los ítems que formarán las versiones cortas de los instrumentos.

Nota. Los criterios presentados en esta figura integran las fuentes de información utilizadas en investigaciones anteriores (e.g., Alcaraz et al., 2013, Maloney et al., 2011; Marsh et al., 2005, Moore et al., 2015). $D T=$ desviación típica; ESEM = Modelo exploratorio de ecuaciones estructurales; $\mathrm{AFC}=$ análisis factorial confirmatorio.

este sentido, Moore et al. (2015) plantearon un procedimiento en el que inicialmente cada miembro del equipo de investigación revisaba individualmente los ítems, para seleccionar aquellos ítems que mejor representaran el constructo (i.e., criterios teóricos), que estuvieran redactados de forma más clara (i.e., criterios de comprensión) y que no fueran redundantes. Y, a continuación, las personas del equipo de investigación ponían en común esta información y discutían hasta consensuar qué ítems debían seleccionar para la versión corta. En el método MIMO, en la Fase I proponemos seguir el procedimiento de Moore et al., pero con la inclusión de criterios psicométricos que complementen los criterios relacionados con el contenido de los ítems. Fruto de este proceso, podremos obtener evidencia relacionada con la validez de contenido (AERA et al., 2014). 


\section{Personas involucradas en esta Fase}

En esta Fase I, confeccionaremos el equipo de investigación que será el responsable de aplicar la totalidad del método MIMO. A nuestro entender, para la creación de este grupo se deben tener en cuenta tres consideraciones. En primer lugar, recomendamos incluir personas familiarizadas con los constructos de interés (Moher et al., 2015; Alcaraz et al., 2013). En segundo lugar, consideramos necesario incluir personas que tengan experiencia en el desarrollo de cuestionarios y de versiones cortas. Asimismo, será recomendable contar con personas expertas en la utilización de metodologías de datos cualitativos que puedan ayudar a preparar, desarrollar y analizar las entrevistas cognitivas y los grupos de discusión de la Fase III. Por último, sugerimos que en el equipo haya personas involucradas en la investigación básica, la investigación aplicada y/o el asesoramiento, de forma que puedan desarrollar una versión corta que pueda solventar los retos planteados en cada uno de estos ámbitos (ver Horvath y Röthling, 2018).

\section{El caso del DCCO-A}

En nuestro caso, para la reducción del DCCQ-A (De Brandt et al., 2018), contamos con un amplio equipo de investigadores e investigadoras pertenecientes al Grup d'Estudis de Psicologia de l'Esport (GEPE). Concretamente, el equipo que participó en el desarrollo de la versión corta estuvo formado por nueve personas (cuatro mujeres y cinco hombres), incluyendo cuatro investigadores seniors, cuatro estudiantes de doctorado y una estudiante de máster. Todas las personas del grupo estaban familiarizadas con las variables evaluadas por el DCCQ-A y con los procedimientos de recogida de datos cualitativos. Complementariamente, cuatro de los investigadores tenían amplia experiencia en el desarrollo y validación de cuestionarios y versiones cortas. Tal como se indica en el método MIMO, este equipo participó tanto en la Fase I como en resto de fases.

Para la selección de los ítems, tuvimos en cuenta los tres tipos de criterios referidos anteriormente. Por ejemplo, en referencia a los criterios teóricos, consideramos las competencias para la carrera dual que habían sido identificadas como más relevantes en investigaciones anteriores (e.g., Miró, Torregrossa, Regüela, Perez-Rivases, y Ramis, 2017; Perez-Rivases et al., 2020). En relación con los criterios psicométricos, pudimos contar con argumentos basados en nuestros propios datos, fruto de investigaciones previas con la versión original del cuestionario. En ese sentido, tuvimos en cuenta la carga factorial de los ítems y su aportación al ajuste de los modelos de medida, entre otros criterios psicométricos. Por último, como criterios de comprensión de los ítems, examinamos, por ejemplo, la longitud de los ítems y que expusieran con claridad qué competencia estaban evaluando. Para cada uno de los ítems, pusimos en común toda la información recopilada y decidimos sobre su selección o eliminación. Como resultado de esta Fase I, consensuamos la eliminación de 9 ítems, quedándonos con una versión de 20 ítem y 5 ítems por factor.

\section{Fase II: Panel de personas expertas}

\section{Desarrollo de la Fase II}

En esta segunda fase, proponemos invitar a un panel de personas expertas que nos ayude a refinar la primera selección de ítems realizada en la Fase I. Así, la inclusión de un panel de personas altamente conocedoras de la temática permitirá evaluar el grado en que los ítems 
seleccionados representan el dominio de interés. Además, el panel de personas expertas ayudará a disminuir el sesgo en la selección de ítems (Boateng et al., 2018). Por último, al igual que en la fase anterior, los procedimientos de esta fase permitirán disponer de evidencia relacionada con la validez de contenido (AERA et al., 2014).

Para llevar a cabo esta fase, en primer lugar, proponemos contactar con las personas que potencialmente podrían formar parte del panel. Una vez estas personas acepten colaborar en el estudio, se les enviará una breve descripción de las medidas a evaluar, así como las instrucciones para llevar a cabo el proceso de selección de ítems. En ese sentido, en línea con el estudio de Milavic et al. (2019), se les podría pedir que seleccionaran aquellos cuatro ítems de cada escala que mejor representaran el constructo. Complementariamente, recomendamos solicitarles que evalúen el contenido de los ítems (e.g., relevancia), tal como se suele pedir en los artículos que desarrollan y validan cuestionarios (e.g., Hill, MacNamara, y Collins, 2019), y que aporten retroalimentación cualitativa para completar sus evaluaciones (e.g., Hoffmann y Loughead, 2019). Una vez recogidas sus impresiones, el equipo de investigación será el encargado de analizar el grado de acuerdo entre personas expertas, calculando el porcentaje de concordancia (e.g., Milavic et al., 2019) o estadísticos específicos, como el coeficiente kappa de Cohen (ver Boateng et al., 2018). Tal como explican Hoffmanny Loughead (2019), la retroalimentación ofrecida por las personas expertas también aportará valiosa información sobre qué ítems deben ser retenidos, revisados o eliminados de la versión corta. Fruto de esta Fase II, se obtendrá la primera versión corta (Versión 1) del cuestionario que se esté trabajando.

\section{Personas involucradas en esta Fase}

Es necesario tener en cuenta que las personas que formen parte del panel de personas expertas deben ser independientes del equipo de investigación (Boateng et al., 2018). De esta forma, sus respuestas no se verán influenciadas por los argumentos del equipo de investigación. En cuanto al número, habitualmente se han utilizado entre cinco y siete personas expertas (ver Haynes, Richard, y Kubany, 1995), aunque otras investigaciones hayan optado por incluir una cantidad mayor (Milavic et al., 2019). En cualquier caso, tal como señalan Boateng et al. (2018), incrementar el número de personas expertas ayudará a incrementar también la robustez de sus evaluaciones.

\section{El caso del DCCQ-A}

Para esta segunda fase, contamos con la colaboración de cuatro expertas y dos expertos que no formaban parte del equipo de investigación. Todas estas personas tenían experiencia tanto en la investigación aplicada como en el asesoramiento en psicología del deporte y trabajaban habitualmente en una universidad (dos personas), un centro de alto rendimiento (una persona), o en clubes con servicios de apoyo a la carrera dual (tres personas). Por lo tanto, todas ellas tenían un amplio conocimiento sobre la gestión de la carrera dual en deportistas enfocados al rendimiento.

Una vez aceptaron participar en el estudio, la tarea que les encomendamos fue doble. Por un lado, les pedimos que evaluaran en una escala de 1 a 5 la relevancia de cada uno de los ítems. Por el otro, les pedimos que indicaran qué ítems creían que debían ser eliminados y 
las razones por las que lo consideraban. Complementariamente, les dimos la posibilidad de aportar sugerencias que mejoraran la comprensión de los ítems.

A continuación, nuestro equipo de investigación se encargó de centralizar las respuestas del grupo de personas expertas en un único documento. El análisis de estas respuestas nos permitió identificar los ítems que debían ser eliminados, en base a cuatro criterios que aplicamos en el siguiente orden: (1) la concordancia en los ítems que proponían eliminar, en porcentaje, (2) las puntuaciones medias más bajas en cuanto a la relevancia otorgada a cada ítem, (3) la menor desviación típica en las puntuaciones medias de la relevancia otorgada a cada ítem, y (4) los argumentos aportados por las personas expertas justificando la eliminación de los ítems. Como resultado del análisis de las respuestas del panel de personas expertas, redujimos el cuestionario de 20 a 16 ítems, con cuatro ítems por factor (Versión 1).

\section{Fase III: Compresión por parte de la población diana}

\section{Desarrollo de la Fase III}

La Fase III permite asegurar que el contenido de los ítems incluidos en la Versión 1 es significativo y comprensible para las personas de la población diana. En este sentido, los procedimientos de esta fase permiten obtener evidencia de la validez relacionada con el proceso de respuesta (AERA et al., 2014). Según señalan Boateng et al. (2018), pre-evaluar el cuestionario con la población diana ayuda a minimizar la mala comprensión de los ítems y a reducir el consecuente error de medida. Complementariamente, los procedimientos de esta fase permiten adaptar los ítems al contexto de la población diana (e.g., alto rendimiento), lo cual ayudará a que los ítems tengan sentido para estas personas (Horvath y Röthlin, 2018).

De acuerdo con Boateng et al. (2018), las entrevistas cognitivas son la herramienta más recomendada para incluir la perspectiva de las personas que forman parte de la población objetivo. Concretamente, las entrevistas cognitivas permiten comprobar que los ítems se entienden y que recogen la información pretendida por las personas que han desarrollado el instrumento (Beatty y Willis, 2007). En base a los resultados de las entrevistas cognitivas, los ítems pueden ser ligeramente modificados o clarificados para que recojan los contenidos esperados.

Complementariamente, hay otras herramientas que pueden ser de utilidad para recoger las impresiones de la población diana. En ese sentido, recomendamos la utilización de grupos de discusión (e.g., Moore et al., 2015) que puedan completar la información recogida en las entrevistas cognitivas. Estos grupos de discusión son especialmente útiles para obtener formas alternativas de redactar los ítems, en el caso de que alguno de ellos no sea del todo comprensible para la población diana (e.g., Ramis, Torregrossa, Viladrich, y Cruz, 2010).

Como cierre de esta fase, el equipo de investigación tiene la tarea de reunir y analizar la información recogida en las entrevistas cognitivas y los grupos de discusión. En ese sentido, recomendamos incluir aquellas propuestas de redactado que mejoren la comprensión de los ítems sin modificar el significado de éstos. Como resultado de esta Fase III, se obtendrá la segunda versión corta (Versión 2) del cuestionario que se esté reduciendo. 


\section{Personas involucradas en esta Fase}

Para desarrollar los procedimientos de la Fase III, será necesario contar con personas que formen parte de la población diana. En este sentido, recomendamos identificar aquellos colectivos que puedan tener más dificultades de comprensión (e.g., deportistas más jóvenes) para asegurarse que quedan representados en las entrevistas cognitivas y los grupos de discusión. De acuerdo con el planteamiento de Boateng et al. (2018), se deberían llevar a cabo 5-15 entrevistas cognitivas, en dos o tres rondas hasta llegar a la saturación de la información (i.e., cuando no emergen nuevas ideas en las siguientes entrevistas). En cuanto al número de participantes en los grupos de discusión, Alcaraz et al. (2013) condujeron dos grupos de discusión con un total de 17 participantes.

\section{El caso del DCCO-A}

Para la evaluar la comprensión de los ítems por parte de personas pertenecientes a nuestra población diana (i.e., deportistas enfocados al rendimiento que estaban viviendo una carrera dual), condujimos tanto entrevistas cognitivas como grupos de discusión. Concretamente, las personas participantes en esta fase compartían edad (i.e., mayores de 14 años), la compaginación de los estudios con el deporte enfocado al rendimiento y se encontraban en una categoría previa a vivir la transición de júnior a sénior.

En primer lugar, en las entrevistas cognitivas, administramos individualmente la Versión 1 del DCCQ-A a deportistas y les pedimos que verbalizaran el proceso cognitivo que habían seguido para ofrecer sus respuestas (Beatty y Willis, 2007). De esta forma, pudimos determinar si las personas entendían los ítems y si respondían de una forma que reflejara su experiencia (Boateng et al., 2018). A continuación, reunimos a las personas que habían participado en las entrevistas cognitivas en grupos de 5 o 6 personas para desarrollar los grupos de discusión. En éstos, les pedimos a las personas participantes que compartieran con el grupo las dudas que hubieran tenido y tomamos nota tanto de éstas como de las propuestas de modificación que se generaban del intercambio entre deportistas y miembros del equipo de investigación. Además de las dudas expresadas directamente por los y las deportistas, les preguntamos por conceptos que pudieran ser confusos, como por ejemplo "ser asertivo/a". Como último paso, el equipo de investigación analizó la información recogida e introdujo pequeños cambios en los ítems que recogieran las sugerencias de las personas de la población diana. Así, gracias a las entrevistas cognitivas y a los grupos de discusión, pudimos pulir la versión corta del DCCQ-A, asegurando que los y las participantes potenciales comprendían sus ítems de la forma esperada.

\section{Fase IV: Evaluación de la versión corta'}

\section{Desarrollo de la Fase IV}

En la cuarta y última fase, proponemos realizar las distintas recogidas de datos necesarias para finalizar la validación de las versiones cortas. Los análisis de los datos obtenidos permitirán disponer de evidencia relacionada con la validez de estructura interna y de relación

\footnotetext{
${ }^{1}$ Para presentar la Fase IV decidimos explicar únicamente su desarrollo, sin vincularla con el ejemplo de la versión corta del DCCQ-A. Haber desarrollado el ejemplo (e.g., análisis de datos, resultados obtenidos) habría alargado innecesariamente el artículo con información que no estaría dando respuesta al objetivo de la presente investigación (i.e., describir el procedimiento del método MIMO).
} 
con otras variables (AERA et al., 2014). En ese sentido, nos alineamos con el planteamiento de Boateng et al. (2018), que proponen evaluar psicométricamente la escala mediante tres pasos que analizan la dimensionalidad (i.e., testear si las variables latentes son las hipotetizadas), la fiabilidad y la validez de los instrumentos desarrollados. Complementariamente, de acuerdo con las recomendaciones de Marsh et al. (2005) para el desarrollo de versiones cortas, será necesario comprobar que las diferencias de género y edad son consistentes entre la versión corta y el cuestionario original. Así, de acuerdo con las propuestas de Boateng et al. y Marsh et al., empezaríamos por realizar los análisis factoriales confirmatorios, el análisis de invariancia y el estudio de la consistencia interna de las escalas.

Los resultados de estos análisis permitirán estimar si la versión corta puede darse como satisfactoria. En el caso de que alguno de los ítems haya mostrado un rendimiento pobre, el equipo de investigación debe analizar la posibilidad de introducir algún cambio (e.g., Alcaraz et al., 2013). Por último, será necesario obtener evidencia vinculada con la validez de relación con otras variables. En este sentido, se debe comprobar que las versiones cortas muestran las mismas evidencias de validez convergente y divergente que la versión original (Horvath y Röthlin, 2018).

\section{Personas involucradas en esta Fase}

En esta fase será necesario definir la población diana que contestará las versiones cortas, la cual dependerá de cada investigación. En este sentido, deberemos anticipar el tamaño de muestra que requiere nuestro estudio. De acuerdo con la revisión de Boateng et al. (2018), no existe un criterio universal que sea aplicable a todos los procesos de desarrollo de cuestionarios. En general, se suele recomendar una ratio de diez participantes por cada ítem incluido en el cuestionario, o un tamaño de muestra de como mínimo 300 participantes (ver Boateng et al.). De forma complementaria a estos criterios, a la hora de anticipar el tamaño de la muestra, será también recomendable contar con una muestra heterogénea, que recoja la variabilidad existente en la población objetivo (Clarke y Watson, 1995). Paralelamente, en esta fase también participará el equipo de investigación, tanto para el análisis de datos como para trabajar en el refinamiento de la versión corta, en el caso de que fuera necesario.

\section{Conclusiones}

En el presente estudio hemos presentado el método de Máxima Información, Mínima Molestia (MIMO) para desarrollar y validar versiones cortas de cuestionarios ya existentes en el campo de la psicología del deporte (ver Figura 1 y Figura 2). Concretamente, el MIMO se presenta como un método basado en la evidencia que permite disminuir la carga de las personas participantes y que, en consecuencia, puede ayudar a realizar procesos de evaluación más eficientes. En este artículo, también hemos ofrecido la justificación para cada una de las cuatro fases del modelo, así como una propuesta de desarrollo y un ejemplo para ayudar a las personas lectoras a comprender cómo aplicar el método MIMO. 
Tal como se ha presentado a lo largo de las cuatro fases, el método MIMO propone incorporar distintas fuentes de información en el desarrollo de las versiones cortas, incluyendo: (a) investigaciones previas (criterios teóricos y psicométricos, Fase I), (b) el equipo de investigación (Fases I a IV), (c) el panel de personas expertas (Fase II), y (d) personas pertenecientes a la población diana (Fase III). De esta forma, la versión corta desarrollada mediante el método MIMO estará en consonancia con la literatura sobre los constructos de interés, podrá satisfacer a las personas que utilicen el instrumento (i.e., personas involucradas en investigación básica, investigación aplicada y asesoramiento), y será comprendida por las personas que lo contesten. A nuestro entender, este enfoque refuerza el proceso de creación de versiones cortas, dado que permite disponer de distintas fuentes que apoyen las propiedades psicométricas de la versión corta resultante.

Concretamente, gracias al desarrollo del método MIMO, se obtienen evidencias relacionadas con distintos tipos de validez, tal como recomiendan los Estándares de la AERA et al. (2014). Complementariamente, las versiones cortas desarrolladas con este método satisfacen los criterios indicados en cuanto al contenido de los ítems, la estructura factorial, la fiabilidad y la relación con otras variables (Maïano et al., 2008; Marsh et al., 2005; Smith et al. 2000). Por último, el método MIMO también cumple con la recomendación de Horvath y Röthlin (2018) de que los instrumentos utilizados en la investigación aplicada y el asesoramiento no sean únicamente válidos y fiables, sino también cortos y de gran usabilidad. En esta línea, el método propuesto puede ayudar a tender puentes entre la investigación básica y la práctica aplicada, facilitando que los instrumentos desarrollados en la primera puedan ser más fácilmente utilizados por las personas profesionales que asesoran a los deportistas y a los otros agentes involucrados en el deporte.

En resumen, el método MIMO ofrece una forma sistemática de reducir la longitud de los cuestionarios en el ámbito de la psicología del deporte. Concretamente, este método permite desarrollar versiones cortas eficientes que cuiden de las personas participantes, reduciendo el tiempo y los recursos que éstas invierten, al mismo tiempo que permiten capturar los aspectos clave de los constructos de interés, minimizan las pérdidas de fiabilidad y validez asociadas tradicionalmente a la reducción instrumentos (e.g., Smith et al., 2000), y permiten incrementar la calidad de los datos recogidos.

\section{Referencias}

Aiken, L. R. (1985). Three coefficients for analyzing the reliability and validity of ratings. Educational and Psychological Measurement, $45,131-142$.

Alcaraz, S., Viladrich, C., y Torregrosa, M. (2013). Less time, better quality. Shortening questionnaires to assess team environment and goal orientation. Spanish Journal of Psychology, 16(E77), 1-14.

American Educational Research Association (AERA), American Psychological Association (APA), y National Council on Measurement in Education (NCME) (2014). The Standards for Educational and Psychological Testing. Washington, DC: American Psychological Association.

Beatty, P. C. y Willis, G. B. (2007). Research synthesis: the practice of cognitive interviewing. Public Opinion Quarterly, 71, 287-311. 
Boateng, G. O., Neilands, T. B., Frongillo, E. A., Melgar-Quiñonez, H. R., y Young, S. L. (2018). Best Practices for developing and validating scales for health, social, and behavioral research: A Primer. Frontiers in Public Health, 6(149), 1-18.

Clarke, L. A. y Watson, D. (1995). Constructing validity: basic issues in objective scale development. Psychological Assessment, 7, 309-319.

Coste J., Guillemin F., Pouchot J., y Fermanian J. (1997). Methodological approaches to Shortening Composite Measurement Scales. Journal of Clinical Epidemiology, 50, 247-252.

De Brandt, K., Wylleman, P., Torregrossa, M., Schipper-van Veldhoven, N., Minelli, D., Defruyt, S., y de Knop, P. (2018). Exploring the factor structure of the Dual Career Competency Questionnaire for Athletes in European pupil- and student-athletes. International Journal of Sport and Exercise Psychology, 35(S1), 1-18.

Haynes, S. N., Richard, D. C. S., y Kubany E. S. (1995). Content validity in psychological assessment: a functional approach to concepts and methods. Psychological Assessment, 7, 238-247.

Hill, A., MacNamara, A., y Collins, D. (2019). Development and initial validation of the Psychological Characteristics of Developing Excellence Questionnaire version 2 (PCDE02). European Journal of Sport Science, 19(4), 517-528.

Hoffmann, M. D., y Loughead, T. (2019). Preliminary development of a questionnaire to assess peer athlete mentoring functions: the Athlete Mentoring Questionnaire (AM0). Measurement in Physical Education and Exercise Science, 23(1), $10-25$.

Horvarth, S., y Röthlin, P. (2018). How to improve athletes' return of investment: Shortening questionnaires in the applied sport psychology setting. Journal of Applied Sport Psychology, 30(2), 241-248.

Jokovic A., Locker D., y Guyatt G. (2006). Short forms of the Child Perceptions Questionnaire for 11-14-year-old children (CPQ11-14): Development and initial evaluation. Health and Quality of Life Outcomes, 4(4). https://doi.org/10.1186/14777525-4-4

Little, T. D., Jorgensen, T. D., Lang, K. M., y Moore, E. W. G. (2014). On the joys of missing data. Journal of Pediatric Psychology, 39(2), 151-162.

Maïano, C., Morin, A. J. S., Ninot, G., Monthuy-Blanc, J., Stephan, Y., Florent, J.-F., y Vallée (2008). A short and very short form of the physical self-inventory for adolescents: Development and factor validity. Psychology of Sport and Exercise, 9, 830-847.

Maloney, P., Grawitch, M. J., y Barber, L. K. (2011). Strategic item selection to reduce survey length: Reduction in validity? Consulting Psychology Journal: Practice and Research, 63(3), 162-175.

Marsh, H. W., Ellis, L., Parada, R., Richards, G. E., y Heubeck, B. (2005). A short version of the Self-Description Questionnaire II: Operationalizing criteria for short-form evaluation with new applications of confirmatory factor analyses. Psychological Assessment, 17, 81-102.

Milavic, B., Padulo, J., Grgantov, Z., Mili , M., Mannarini, S., Manzoni, G. M., ... Rossi, A. (2019). Development and factorial validity of the Psychological Skills Inventory for Sports, Youth Version - Short Form: Assessment of the psychometric properties. PLoS One, 14(8), e0220930.

Miró, S., Torregrossa, M., Regüela, S., Pérez-Rivases, A., y Ramis, Y. (2017). Competencias para la planificación de la carrera dual de deportistas de alto rendimiento. Revista de Psicología del Deporte, 26(S4), 28-33.

Moore, E. W., Brown, T. C., y Fry, M. D. (2015). Psychometric properties of the Abbreviated Perceived Motivational Climate in Exercise Questionnaire. Measurement in Physical Education and Exercise Science, 19, 186-199.

Mülhan H., Bullinger M., Power M., y Schmidt S. (2008). Short forms of subjective quality of life assessments from cross- cultural studies for use in surveys with different populations. Clinical Psychology and Psychotherapy, 15, 142-153.

Noetel, M., Ciarrochi, J., Sahdrab, B., y Lonsdale, C. (2019). Using genetic algorithms to abbreviate the Mindfulness Inventory for Sport: A substantive-methodological synthesis. Psychology of Sport \& Exercise 45, 101545. https://doi. org/10.1016/j.psychsport.2019.101545

Perez-Rivases, A., Pons, J., Regüela, S., Viladrich, C., Pallarès, S., y Torregrossa, M. (2020). Spanish female student-athletes' perception of key competencies for successful dual career adjustment. International Journal of Sport and Exercise Psychology. Manuscrito aceptado para publicación.

Ramis, Y., Torregrossa, M., Viladrich, C., y Cruz, J. (2010). Adaptación y validación de la versión española de la Escala de Ansiedad Competitiva SAS-2 para deportistas de iniciación. Psicothema, 22(4), 1004-1009. 
Smith G. T., McCarthy D. M., y Anderson K. G. (2000). On the sins of short-form development. Psychological Assessment, 12 , 102-111.

Vlachopoulos, S. P., Leptokaridou, E. T., y Fox, K. R. (2014). Development and initial evidence of validity of a short form of the physical self-perception profile for Greek adults. International Journal of Sport and Exercise Psychology, 12(2), 166-184.

Watson D., y Clark L. A. (1997). Measurement and mismeasurement of mood: Recurrent and emergent issues. Journal of Personality Assessment, 68, 267-296.

Yan, T., Fricker, S., y Tsai, S. (2020). Response burden: What is it and what predicts it? En P. Beatty, D. Collins, L. Kaye, J. L. Padilla, G. Willis y A. Wilmot (Eds.), Advances in questionnaire design, development, evaluation and testing (pp. 193-212). Hoboken, NJ: John Wiley \& Sons.

\section{Agradecimiento:}

"Este trabajo se ha realizado gracias a la subvención RTI2018-095468-B-100 del Ministerio de Ciencia, Innovación y Universidades."

"Los autores y autoras de este manuscrito quieren agradecer a Marina García, José Tomás Mejías y Rocío Zamora su colaboración continuada en esta investigación." 